\title{
L'environnement et le développement : enjeux socio-politiques à l'échelle locale. Premières approches de la fabrication des villes et des vies durables, à Florianópolis (Brésil)
}

\author{
Clara Rosana Chagas Paraboa \\ Doctorante en Anthropologie \\ Faculté d'Anthropologie et de Sociologie, Université Lumière Lyon 2 \\ Dirección electrónica: cparaboa@yahoo.com.br
}

Chagas Paraboa, Clara Rosana (2016). "L'environnement et le développement : enjeux socio-politiques à l'échelle locale. Premières approches de la fabrication des villes et des vies durables, à Florianópolis (Brésil)". En: Boletín de Antropología. Universidad de Antioquia, Medellín, vol. 31, N. ${ }^{\circ}$ 51, pp.210-233. DOI: http://dx.doi.org/10.17533/udea.boan.v31n51a11

Texto recibido: 28/11/2014; aprobación final: 12/10/2015

Résumé. Le développement durable, nouveau paradigme environnemental, touche à peine les pays du sud. Les choix des modèles urbains sont encore posés sur les dimensions économiques, puis « écologiques » et en dernier lieu sociales. Les enjeux politiques et financiers sont importants dans les espaces encore préservés dans la ville ainsi que pour ceux qui y vivent. Cet article présente une première étude ethnographique sur un projet collectif socio-environnemental issu d'une communauté scolaire au sud du Brésil. L'école joue un rôle essentiel dans l'évolution des pratiques démocratiques participatives et dans la protection du patrimoine naturel et socioculturel local, ainsi que l'engagement politique de l'anthropologue.

Mots-clés : anthropologie de l'environnement, développement durable, participation démocratique, aménagement de la ville, communauté scolaire.

\section{Medio ambiente y desarrollo: cuestiones socio-políticas locales. Un estudio preliminar del desarrollo de las ciudades y vidas sostenibles, en Florianópolis (Brasil)}

Resumen. El desarrollo sostenible, nuevo paradigma ambiental, apenas toca los países del sur. Las elecciones de los modelos urbanos todavía se colocan en problemáticas en primer lugar económi- 
cas, segundo ambientales y por último sociales. Los intereses políticos y económicos son importantes para los espacios conservados de la ciudad y para los que viven allí. Este trabajo presenta un estudio etnográfico de un proyecto de grupo socio-ambiental de la comunidad escolar en el sur de Brasil. La escuela juega un papel vital en la evolución de las prácticas democráticas participativas y la protección del patrimonio natural y socio-cultural local, así como el compromiso político del antropólogo.

Palabras-clave: antropología del medio ambiente, desarrollo urbano sostenible, participación democrática, planificación de la ciudad, comunidad escolar.

\section{Environment and development in local socio-political issues. Preliminary studies about the development of sustainable cities and life, in Florianópolis (Brazil)}

Abstract. Sustainable development, a new environmental paradigm, influences slightly the southern countries. The choices of urban models are still placed on the economic at first, then the environmental and latter on the social. Political and financial stakes are important for preserved areas in cities and also for those who live there. This paper presents a preliminary ethnographic study of a socio-environmental project from a school community in southern Brazil. The school plays a vital role in the evolution of participatory democratic practices and the protection of natural and local socio-cultural heritage, as well as the political commitment of the anthropologist.

Key-words: environmental anthropology, sustainable development, democratic participation, urban planning, school community.

\section{Meio ambiente e desenvolvimento: questões sociopolíticas locais. Um estudo preliminar do desenvolvimento das cidades e vidas sustentáveis, em Florianópolis (Brasil)}

Resumo. O desenvolvimento sustentável, novo paradigma ambiental, somente toca os países do sul. As eleições dos modelos urbanos ainda se colocam em problemáticas em primeiro lugar econômicas, segundo ambientais e no final sociais. Os interesses políticos e econômicos são importantes para os espaços conservados da cidade e para os que moram ali. Este trabalho apresenta um estudo etnográfico de um projeto de grupo socioambiental da comunidade escolar ao sul do Brasil. A escola desempenha um papel muito importante na evolução das práticas democráticas participativas e na proteção do patrimônio natural e sociocultural local, mesmo que o compromisso político do antropólogo.

Palavras-chave: antropologia do meio ambiente, desenvolvimento urbano sustentável, participação democrática, planejamento da cidade, comunidade escolar.

\section{Introduction}

L'approche de l'anthropologie environnementale n'est plus restreinte à des espaces naturels ou exotiques ni à la quête de l'influence de la nature sur la culture des peuples lointains et vice-versa, selon des perspectives issues de modèles liés au paradigme déterministe écologique. Cette branche de l'anthropologie, l'anthropologie environnementale, est de plus en plus préoccupée par l'analyse et l'interprétation des multiples modes d'interaction des sociétés avec leur environnement, notamment 
dans les espaces urbains, et qui révèlent différentes conceptions de la nature et de l'environnement.

Les projets d'éco-villages, d'éco-quartiers, de mobilité urbaine douce, de choix alimentaires, de jardins urbains, de pratiques énergétiques, entre autres, sont des exemples à retenir dans le changement du rapport entre la culture urbaine, la nature et l'environnement. Toutefois, bien que ces nouveaux concepts pour les villes durables soient louables, Faburel (2012:15) met en lumière dans son article " La ville durable... ", l'écart entre discours officiels et pratiques d'urbanisme ainsi que l'aménagement durable au constat des ségrégations socio-environnementales.

Ces projets sont surtout prédéfinis par des responsables municipaux par le biais de pratiques de concertation urbaine qui prennent peu en compte les desseins et les vraies nécessités des habitants. Dans cet article, il s'agit de présenter une étude ethnographique réalisée entre 2010 et 2012 dans le cadre d'une recherche doctorale, dans un quartier de la ville de Florianópolis située au sud du Brésil. Notre objectif est de montrer le rapport qu'un groupe social, appartenant à une communauté côtière, entretient avec son environnement, par le biais de projets menés par l'école locale, ainsi que l'investissement dans la définition de leur espace de vie à partir d'une expérience de participation démocratique que nous appelons autonome, à savoir, l'élaboration d'un projet qui définit l'occupation du sol dans les environs de l'école pour la protection du patrimoine naturel et socio-culturel local.

Ainsi, à partir d'observations participantes, d'interviews non-structurées et documents divers, nous avons analysé les discours, les pratiques des acteurs et les enjeux liés à un projet audacieux mené par la communauté scolaire. ${ }^{1}$ Le projet Entorno Escolar, soutenu par diverses associations existantes dans la région, prévoit entre autres la création d'un Corridor écologique, un parc pour protéger l'écosystème local, la conservation d'une maison d'architecture coloniale pour la constitution d'un musée pour la sauvegarde de la culture locale, ainsi que l'expansion de l'école locale et sa transformation en un Institut éducationnel spécialisé en éducation environnementale. Ce projet considéré dans un ensemble de projets locaux, notamment ceux issus du Plan participatif d'urbanisation local, défient les règles et les pratiques actuelles de l'urbanisme municipal projeté pour développer le tourisme international d'élites dans la ville, peut être situé dans une pratique de participation démocratique autonome et dès lors dans une logique de contre-pouvoir (Bacqué, 2005).

1 La communauté scolaire est formée par les familles des élèves, des élèves eux-mêmes, des enseignants, des spécialistes et des personnels en général. Mais ce sont surtout les leaders-membres du Conseil délibératif d'école (CDE) et de l'Association de parents d'élèves et professeurs (APP), donc des organisations institutionnalisées par le Ministère de l'Education et de la Culture, qui ont participé directement aux négociations avec les autorités municipales. 
Dans ce cadre, nous interrogeons l'expérience de participation démocratique (Dewey, 2010; Zask, 2011) à l'heure de la ville durable (Faburel, 2012). D'autre part, nous réfléchissons sur le rôle de l'école publique dans la protection de l'environnement local ainsi que sur l'engagement ethnographique (Cefaï, 2010) dans la quête de la justice socio-environnementale.

L'anthropologie de l'environnement urbain

A l'heure de la mondialisation, les risques environnementaux identifiés dans la réalité climatique de la Planète et dans l'épuisement des ressources naturelles ainsi que dans les discours, idéologies et représentations diverses, sont de plus en plus présents dans les projets de vie des mondes locaux. Etant un sujet particulièrement sensible, l'environnement est devenu un enjeu hautement politique et financier.

Ainsi, le savoir anthropologique (Quentin, 2003), aujourd'hui, ne peut pas être conçu en dehors des questions politiques qui guident les interactions entre les humains et leur environnement urbain, sous peine de limiter les interactions entre les humains et la nature à une vision déterministe de deux voies et sans prendre en compte les expériences actuelles des communautés ${ }^{2}$ qui objectivent l'aménagement de leur espace urbain, tout en conservant les éléments naturels avec le but d'améliorer le futur des nouvelles générations.

La question de la recherche sur l'environnement engage non seulement l'analyse de la dynamique éco-systémique des êtres vivants, l'homme étant partie prenante de celui-ci, en interaction avec la nature ou son milieu physique, biologique ou chimique, mais aussi bien d'autres rapports comme leur propre perception et interprétation de l'environnement, catégories issues de l'anthropologie cognitive de Tyler ${ }^{3}$ (Milton, 1997 : 527). A titre d'exemple, dans cette perspective, nous pouvons réfléchir sur les modes de penser, les points de vue, les besoins, les croyances, les actions, etc. des habitants d'une île qui vivent au bord de la mer et conclure qu'ils ne sont pas les mêmes de ceux qui l'habitent à l'intérieur.

Les démarches scientifiques actuelles de l'anthropologie réfléchissent sur ces aspects, de manière dialectique. Cette diversité culturelle placée dans un contexte plus large d'interactions, doit tenir compte des situations géographique, historique, socioculturelle, politique et économique, celles-ci situées encore dans un processus de mondialisation. Par exemple, on peut supposer que les choix des politiques urbaines, dites durables, pour une métropole continentale comme São Paulo ne sont pas les mêmes que pour une métropole qui est baignée par la mer comme

2 Nous allons employer le terme de «communauté», à l'exception de communauté scolaire, en tant que synonyme de l'ensemble d'habitants d'un quartier, tel qu'il est utilisé à Florianópolis.

3 Selon Milton (1997 : 527), l'anthropologie cognitive se caractérisait pour une approche culturelle dans l'analyse et processus de prise de décision et leurs conséquences socioculturelles, c'est-à-dire une connaissance définie du point de vue de populations étudiées à partir de méthodes adaptées. 
Rio de Janeiro. L'interface culture-nature articule le rapport des habitants avec l'environnement tel que la façon d'habiter le lieu, la manière de se déplacer dans l'espace local, et de vivre les expériences sociales.

Milton (1997 : 532-534) évoque que l'usage et les modes de vie des gens jouent un rôle fondamental dans leur compréhension des catégories, nature et environnement. Celles-ci peuvent être conçues comme séparées de la culture ou comme un continuum ou même les deux en même temps. Selon l'auteur, le discours contemporain des anthropologues sur l'environnement portent sur la fabrication de traits de culture engendrés par l'interaction de l'être humain avec des éléments de son environnement. Ceux-ci, dans un processus dialectique, sont directement liés aux perceptions, aux expériences ainsi qu'aux modes d'appréhension et de conceptualisation, et donc au modèle de comportement adopté envers l'environnement.

Ainsi, peut-on dire que les conceptions de nature se complexifient au gré des interactions que les membres d'une société en particulier tissent avec leur environnement surtout dans les sociétés industrielles. Les groupes de personnes qui pratiquent des multiples modes d'intégration avec leur environnement tels que la culture de la terre et l'élevage extensif, la pêche, l'observation des animaux, la culture de plantes et jardins potagers, les randonnées dans des parcs ou sentiers, le surf, etc., auront sur celui-ci des points de vue multiples et complexes.

Milton (1997 : 535-537) insiste sur l'importance de prendre en compte la perspective anthropologique dans le débat contemporain sur l'environnement, c'est-à-dire, de comprendre la culture locale dans sa diversité, avec ses structures sociales, pratiques quotidiennes, stratégies économiques, idéologies politiques, etc. pour résoudre certains problèmes environnementaux et faciliter la recherche de modes de vie durables.

Cette approche anthropologique implique par cohérence l'engagement de l'anthropologue (Cefaï, 2010) dans l'enseignement, la recherche, dans l'action citoyenne et/ou dans l'expertise auprès d'institutions politiques, judiciaires ou administratives pour la défense des droits des groupes défavorisés ou de territoires, si nécessaire pour améliorer la situation de vie des populations étudiées.

\section{Les paris dans la fabrication des villes et des vies durables}

Les politiques urbaines sont nées d'un idéal économique, social et environnemental. Relativement récentes, elles sont fondées sur la notion de ville durable. Cette notion de ville durable a été développée en 1992 à Rio dans le « Sommet de la Terre » et soutenue par plusieurs pays qui ont adopté un plan d'action pour le Xxi ${ }^{\text {ème }}$ siècle nommé Agenda 21.

Historiquement, la notion de ville durable est une déclinaison de la notion de développement durable dont la première reconnaissance internationale est présente dans la publication du rapport «Halte à la croissance » du Club de Rome en 
1968, lequel rapport a orienté le débat sur l'écodéveloppement dans la conférence de Stockholm en 1972 et puis dans le rapport Brundtland, "Notre avenir à tous ", publié en 1987 par la Commission mondiale sur l'environnement et le développement. Ces notions sont reflétées dans des projets et des lois portés par des collectivités locales ou par des politiques de la ville.

Au Brésil, le Budget participatif initié dans les années 1990 à Porto Alegre, est un exemple remarquable des premières politiques urbaines durables et de participation démocratique dans la ville, suivi par d'autres expériences dans le Brésil, en Amérique Latine (Argentine, Costa Rica et République Dominicaine) et en Europe (Allemagne, Espagne, France et Italie). Au Brésil, d'autres dispositifs de participation présents dans la loi fédérale des politiques urbaines nommée Estatuto da Cidade n'ont pas été configurés comme des pratiques politiques obligatoires dans tout le territoire brésilien avec des méthodes durables pour l'aménagement urbain, car la loi nationale n'est pas souvent respectée dans les projets urbains municipaux.

Le Statut de la Ville 4 réglemente le chapitre «politique de la ville » de la Constitution brésilienne de 1988. Ses principes fondamentaux sont la planification urbaine participative pour le développement de villes durables afin d'éviter ou de corriger les déformations de la croissance urbaine et leurs effets négatifs sur l'environnement. Le texte affirme :

La loi établit des règles d'ordre publique et d'intérêt social qui réglementent l'utilisation de la propriété urbaine en faveur du bien collectif, la sécurité et le bien-être des citoyens et de l'équilibre de l'environnement en garantissant le droit à la ville durable, entendu comme le droit aux réserves foncières pour le futur, au logement, à l'assainissement, aux infrastructures urbaines, au transport et services publics, au travail et au loisir pour les générations présentes et futures. ${ }^{5}$

La problématique de la question urbaine brésilienne est loin d'être résolue, et cela a connu une grande visibilité internationale en 2013 et 2014 avec les manifestations de rue à grande échelle sur tout le territoire brésilien. Mais les problèmes liés à la ville ne peuvent pas être examinés sans qu'on évoque d'abord les mouvements sociaux débutés à la fin de la dictature -des mouvements des années 80- quand le Brésil a traversé la période de l'Ouverture démocratique, ainsi que ceux des années suivantes.

4 Le Statut de la Ville est consolidé par la Loi 10.257 du 10 Juillet 2001. Source: Brasil, Lei Estatuto da Cidade, 2011. http://www.planalto.gov.br/ccivil_03/leis/leis_2001/110257.htm Consulté le $03 / 11 / 2015$.

5 Traduit par nos soins. 
La dictature au Brésil ${ }^{6}$, qui a duré une vingtaine d'années, a eu pour objectif de défendre l'idéologie nationaliste et défendre les valeurs de la tradition, de la famille et de la propriété privée -menacés par l'idéologie des luttes de classes de gauche- au travers du slogan « Brésil Puissance 2000 ».

Selon la philosophe Marilena Chaui (2006 : 41-42) le coup d'état militaire s'est donné trois tâches: l'intégration, la sécurité et le développement national brésilien, dont la «nation» serait consolidée par trois piliers, d'un côté par Dieu et la Nature et d'autre côté par l'Etat. Ceux-ci étaient considérés comme les agents de développement, de modernisation ou grandeur, et une image qui, toujours selon Chaui (2006 : 42), a été construite dans la période coloniale et que s'est conservée même après la proclamation de la République.

L'idéologie tripartie (Dieu, Nature et Etat) constitutive du « verdemarelismo $»^{7}$ brésilien est, à notre avis, présente encore aujourd'hui dans les discours des hommes politiques. On peut même affirmer que cette ligne directrice de la dictature est encore gravée dans les esprits des Brésiliens. Le discours fait par le maire de Florianópolis, suivi du commentaire du directeur du bureau municipal d'habitation de la ville, lors de la présentation du Projet Entorno Escolar dans une audience publique réalisé dans l'amphithéâtre de l'école Dilma Lúcia dos Santos, le 23 février 2008, donne un bel exemple de l'idéologie héritée de la dictature.

[...] Nous sommes privilégiés par la géographie locale, avec les beautés naturelles qui sont très charmantes. Mais nous, au fil du temps, nous sommes en train de détruire tout ce que la nature nous a offert [...] Il n'existe aucun coin de cette ville que ne soit pas merveilleux, n'est-ce pas ? [...] Dans les années 70, il avait environ cent mille habitants à Florianópolis tandis que le Brésil avait environ 90 millions d'habitants. Vous vous souvenez... Je me souviens des années 70, avec la Coupe du Monde au Mexique, quand le Brésil avait été champion, etc. Il avait la chanson 90 millions en action... etc., n'est-ce pas ? Nous étions en Florianópolis cent mille habitants. Le temps est passé. Et aujourd'hui le Brésil a 180... Combien? 189 millions ? Et Florianópolis à quatre cent mille. [...] Cette ville dans les derniers 20,30,40, 50 ans..., enfin, depuis qu'elle a été fondée, a connu une croissance sans la participation du peuple, n'est-ce pas ? Elle n'a pas grandi à travers les gens, elle ne disposait pas d'un plan d'urbanisme, elle n'a pas eu de projet, elle n'a pas eu de planification, n'est-ce pas? Elle s'est élargie à tous les coins et recoins de notre merveilleuse île sous une forme complètement désorganisée, sans contrôle du pouvoir public local,

6 La dictature brésilienne s'insère dans le contexte des dictatures militaires de la seconde moitié xxème siècle dans l'Amérique Latine et soulève deux types principaux d'expressions de résistance: les organisations clandestines (guérillas et les partis communistes ou socialistes) et les manifestations publiques contre le pouvoir autoritaire et contre les restrictions aux droits civils et politiques, en général réprimées par l'armée.

7 L'idéal vert-jaune, couleurs présentes dans le drapeau brésilien, permettait au gouvernement dictatorial de mettre en lumière en tant que « spécificité nationale » les richesses des ressources naturelles du pays, supposées inépuisables, la docilité du peuple, ainsi que le « mérite développementiste » et dès lors la croissance et le progrès du pays. 
régional ou fédéral. Et aujourd'hui, nous sommes ici présents, préoccupés... Et nous allons payer un prix très lourd pour les erreurs commises dans le passé, car corriger cela n'est pas facile, n'est-ce pas ? [...] Nous sommes ici avec le secrétaire de travaux, avec le Dr Nelson, ingénieur et représentant du Bureau du Logement et d'Assainissement de l'Environnement et le professeur Francisco Pereira da Silva (le directeur de l'Institut de Planification Urbaine de Florianópolis - IPUF). Nous sommes bien représentés ici (en regardant le directeur de l'IPUF) et c'est avec lui que je vais partager la responsabilité et la gestion des étapes suivantes qui devront dorénavant être accomplies, ok ? Pour que je puisse dans un court laps de temps... qui le sait, si Dieu le veut, que j'implante ici l'école de l'environnement [...]

[...] J'ai une grande affection (par cette région) de sorte que nous sommes ensemble dans ce projet. Et que Dieu bénisse cette communauté. Je suis sûr que (elle-la communauté) sera victorieuse! (Pozzobom et Rocha, 2013) ${ }^{8}$

Le maire de la ville en s'adressant au public, traite le sujet de la croissance de la population par le biais d'un hymne de la Coupe du Monde des années 70 « 90 milhões em ação, pra frente Brasil do meu coração ». De plus, pendant que le Brésil vivait en pleine dictature militaire, il joue à notre avis avec la force symbolique de l'Etat autoritaire et de l'image du Brésil, en tant que pays du football, dès lors un point de vue aliéné et aliénant.

Cela révèle une sorte de comparaison entre la puissance des supporters du football à la puissance du peuple dans résolution des problèmes qui concernent les infrastructures de la ville. De plus, il suggère que la croissance du peuple est responsable des malaises que la ville éprouve « le nombre de personnes augmentent et avec cela croissent aussi le nombre de problèmes ", les culpabilisant d'une certaine façon, et de ce fait, il se soustrait de ses responsabilités envers les actions gouvernementales et envers les erreurs politiques, notamment les siennes, lors de son premier mandat en tant que maire de la ville, qu'il était en train de s'achever.

Simultanément, le maire exalte les caractéristiques naturelles de la ville. D'un côté, il ajoute son émerveillement par rapport aux beautés de l'île, c'est une façon de toucher l'inconscient collectif du public, en étalant le « mythe fondateur » du Brésil évoqué par Chaui (2006 : 58), la vision du paradis terrestre. D’autre côté, l'évocation d'un dieu providentiel dans son discours est révélateur d'un autre mythe fondateur, la sacralité de l'histoire en tant que volonté et loi divine sur laquelle repose le pouvoir politique. ${ }^{9}$

8 Extrait du discours du Maire de Florianópolis (le plus long) et du directeur de l'Institut de Planification Urbaine de Florianópolis. Réalisation : Traduit par nos soins.

9 Le pouvoir d'un homme de pouvoir peut être considéré comme une grâce divine. Cela implique une idée précise de la représentation politique : le gouvernant représente Dieu, origine transcendante de tout le pouvoir et décision. On appelle le pouvoir qui appartient à Dieu, un régime théocratique (Chaui, $2006: 82-83$ ). 
Dans la trame discursive, les amalgames inférés sont repérés d'une part (i) dans le cadre de l'enchantement de la nature, (ii) de la faiblesse du pouvoir public face à la population croissante et (iii) du pouvoir omniprésent du privé et de l'économique, constitué dans la ville par les entrepreneurs du secteur l'immobilier. Et d'autre part, dans les questions relatives à la personne du maire en tant que représentant du pouvoir public, ainsi que du pouvoir de Dieu, tous plus ou moins prétendus comme des synonymes dans le message passé.

Ermínia Maricato (2013), créatrice du Ministère des villes du gouvernement du Président Lula, ${ }^{10}$ évoque que les villes brésiliennes ont hérité de l'esclavage une lourde peine, un état patrimonialiste et la généralisation de politiques clientélistes et népotistes. Selon la professeure, étant donné que le marché résidentiel privé légal est hautement spéculatif, une grande partie des villes sont construites de façon illégale ou informelle, en dehors de la législation urbaine. Ainsi, la ville formelle est un simulacre de quelques images du " Premier monde ", à l'image des pays développés « les faces des pièces de monnaie, l'une n'existe pas sans l'autre ».

\section{Contextualisation environnementale de la ville de Florianópolis}

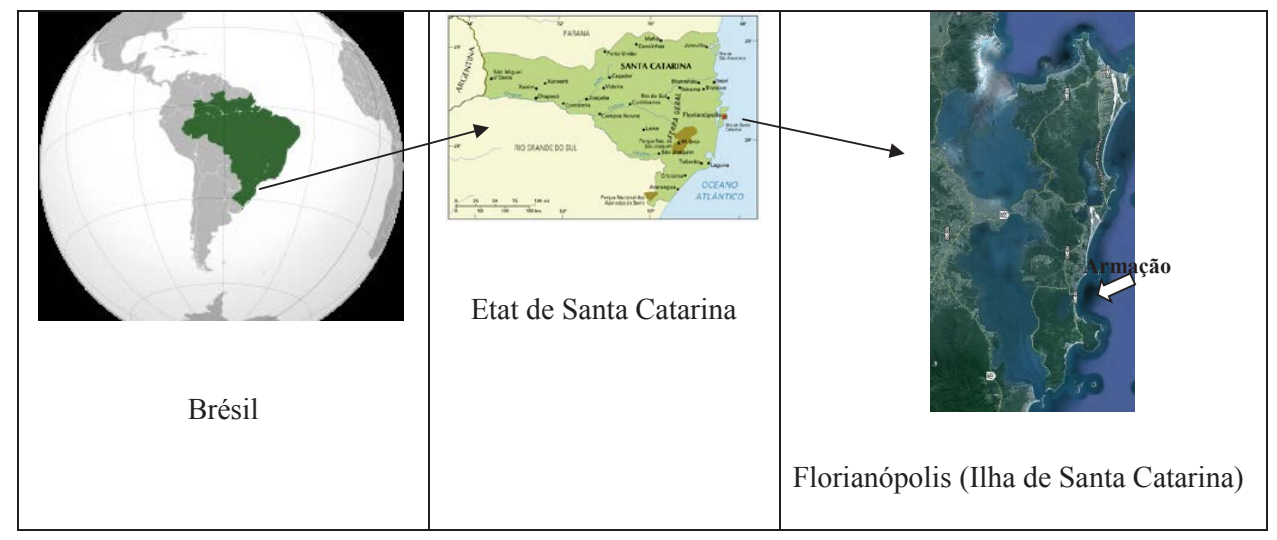

Figure 1. Localisation de Florianópolis et du terrain d'étude. Adapté de Google earth de l'auteu

Florianópolis, ${ }^{11}$ capitale de l'État de Santa Catarina, au sud du Brésil, est située dans une petite partie continentale et son plus grand territoire se trouve dans l'île éponyme -d'environ $54 \mathrm{~km}$ de longueur (nord-sud) et $18 \mathrm{~km}$ de largeur (est-

10 La professeure d'Architecture et urbanisme de l'USP (Université d'Etat de São Paulo), a occupé la fonction de secrétaire exécutive ainsi que de coordinatrice technique de la Politique nationale de développement urbain du Ministère des villes entre 2002 et 2005.

11 Notre terrain de recherche, le quartier d'Armação, est situé au sud de cette ville. 
ouest). La ville est donc essentiellement insulaire, et l'île est surnommée par les autochtones, les étrangers et par l'office de tourisme " L'île de la Magie », grâce non seulement aux légendes issues de la culture locale liées aux sorcières et sorcelleries, mais aussi liées à la beauté et à la fascination des gens par les espaces locaux formés par une géographie séduisante et des écosystèmes naturels envoûtants.

Entre les années 70 à 90 , le pari dans « la vocation naturelle de la ville pour le tourisme $\gg^{12}$ a commencé à donner le ton de l'expansion urbaine. La construction de deux ponts modernes en 1975 et en 1990 a permis le développement du tourisme de masse et l'arrivée d'une grande vague de nouveaux émigrants. L'avènement de l'automobile et la construction des routes goudronnées ont été accompagnés de grands investissements, particulièrement au nord de l'île, dans le secteur immobilier de niveau international, et par conséquent ont accru la valorisation foncière et de l'occupation du sol de manière désordonnée dans l'ensemble de l'ile.

Dans ce contexte, " l'industrie du tourisme » (Lago, 1996 ; Fantin, 2000; Alexandre, 2003) en tant qu'un des principaux piliers du développement et de la croissance économique de la ville, a accéléré le changement du modèle de ville existant. Des remblais ont été réalisés, des mangroves, des dunes et des aires de préservation permanente ont été envahis transformant significativement l'écosystème insulaire.

De nouveaux tracés urbains tels que des avenues et des routes ont contribué à l'édification et à la verticalisation des bâtiments ainsi qu'au rapprochement des sites éloignés comme ceux situés à l'intérieur de l'île ainsi que des plages isolées.

Tout ce changement additionné de l'augmentation pharaonique de la population -de 193530 hab. en 1991, elle passe à 339063 en $2000^{13}$ - a déclenché en continu des problèmes sociaux et environnementaux dans l'île. Et ceux-ci ont fortement contribué au changement de l'essence culturelle et socio-économique héritée de la colonisation açoréenne et de l'environnement de l'île dans les années suivantes. La pêche artisanale a été remplacée par le modèle industriel en changeant le mode de vie des anciens habitants. L'expansion de l'espace urbain a généré un changement de comportement des enfants de pêcheurs et de paysans en faisant se dégrader le sentiment d'appartenance à une communauté. Ces derniers étant allés travailler dans le secteur tertiaire ont contribué au processus de spatialisation et de spécialisation des activités urbaines (Lago, 1996).

Aujourd'hui la ville de Florianópolis peut être présentée quasiment par deux images révélatrices: la première montre un grand nombre de personnes arrivant

12 Voir Fantin, $2000: 16$.

13 Actuellement, la population de la ville est évaluée en environ 469.690 hab. (2015) Source: Ibge, Censo demográfico Informações completas da cidade de Florianópolis. http://cidades.ibge.gov. br/xtras/perfil.php?lang=\&codmun=420540\&search $=\mid$ infogr $\%$ E1ficos:-informa $\%$ E7\%F5escompletas Consulté le 03/11/2015. 
quotidiennement dans la ville -en train de fuir les petites villes «sans avenir» et les grands centres urbains surpeuplés- et ces personnes deviennent les nouveaux acteurs de l'urbanisation chaotique des paysages côtiers qui constituent la ville.

La deuxième expose la transformation des relations établies auparavant entre l'homme traditionnel et la nature, comme c'est le cas des habitants plus anciens, les pêcheurs, les femmes dentelières et les petits agriculteurs. Jusqu'aux années 70, ceux-là vivaient en communautés « isolées » et menaient leurs activités quotidiennes rythmées par la nature et les relations de proximité assez cadrées et familiales.

L'urbanisation imposée par l'expansion de la ville a fini par transformer les aspects de la culture locale ainsi que les relations entre ces personnes. Ces mutations provoquées par la séparation de l'habitat de l'environnement de travail ont été suivies de transformations dans le propre sein de la famille, auparavant centrée dans la production familiale.

Comme nous le rappelle le géographe brésilien Ewerton Machado (2000 : 127), qui a rédigé sa thèse de doctorat sur l'urbanisation de Florianópolis à l'heure de la mondialisation, la production et l'appropriation de l'espace de la ville a été possible grâce aux actions modernisatrices développées principalement par les agents de l'Etat qui voulaient donner à la capitale une allure métropolitaine, ce qui a été fait également dans d'autres capitales brésiliennes dans les années 70 . Ces actions ont permis de créer les infrastructures et les apports du développement urbain dans la capitale, transformée en 1998 en région métropolitaine, comme tremplin pour les activités des entrepreneurs du secteur privé.

Mais, le fait est que, conçue sans une planification urbaine conséquente, affaiblie par l'absence d'infrastructures urbaines et à la défaillance dans la prévision du contingent de personnes qui débarqueraient sur l'île, l'Ile de la Magie a fait le choix d'un modèle d'occupation du sol basé sur l'exclusion des anciens habitants et la ségrégation spatiale.

Le résultat est que l'absence de projet urbain solide a déclenché dans la ville une urbanisation arbitraire et désordonnée. Certes, cela a «bénéficié » aux nouveaux arrivants, issus de différentes classes sociales, dans la possession ou l'acquisition de terres à très faibles prix, mais fondamentalement cela a servi les intérêts privés des grandes sociétés du bâtiment et des travaux publics. Dans ce décor, des espaces de l'île -même ceux préalablement protégés par des lois environnementales ${ }^{14}$ sont transformés et aménagés sans relâche pour accueillir les touristes des classes moyennes-supérieures voire très aisées.

Pendant ce temps-là, les politiques urbaines négligent les besoins basiques d'infrastructure tels que l'assainissement, la collecte des déchets ménagers, l'extension du système de drainage des eaux pluviales, le pavage des rues et la

14 Ici on ne rentrera pas dans le sujet de la manipulation et du changement des lois environnementales dans les dernières années pour faire bénéficier les sociétés du bâtiment et du tourisme. 
construction de trottoirs ainsi qu'un ensemble de services publics : le système de transport en commun, la santé, l'éducation de qualité, des espaces de loisir, etc., pour les classes moins aisées, les classes populaires et les communautés traditionnelles de pêcheurs de l'île. Manifestement, quand il s'agit de choisir entre l'apparat luxueux ${ }^{15}$ et la définition et mise en place de politiques urbaines pour améliorer la vie quotidienne des pauvres, la réalité à Florianópolis est indéniablement arbitraire.

\section{Entre l'environnement et le développement : conflits d'intérêt dans le quartier d'Armação}

Cette localité d'environ trois mille habitants ${ }^{16}$ est située à 25 kilomètres du centre de la ville de Florianópolis. Pour l'administration de la mairie, elle est considérée dans la nomenclature municipale en tant qu'un des sept « quartiers $»^{17}$ existants dans le District Pântano do Sul.

La localité d'Armação, peut être considérée comme une porte de transition entre l'urbain et le rural, " un trésor au pied de l'arc en ciel » pour les communautés traditionnelles qui conservent des pratiques liées à la pêche, l'artisanat et l'agriculture familiale, ainsi que pour ceux qui cherchent à s'éloigner du centre névralgique de la ville, mais également pour ceux qui portent des projets orientés vers le tourisme d'élite et vers des intérêts privés, présents déjà dans d'autres régions de l'île.

Avec un paysage unique et une richesse naturelle, historique et multiculturelle particulière, le quartier d'Armação est l'un des endroits de l'île qui résiste encore au processus de l'étalement urbain. En fait, le sud de la « ville-île » est une des seules régions qui conteste le type de développement lié au tourisme qu'on observe depuis la fin des années 80 entamé dans le centre-ville et étendu à l'est et au nord de l'ile, et qui a crû de façon exponentielle depuis les années 90.

Dans ce cadre de différentiation spatiale, il faut considérer les conditions géographiques et les microclimats de l'ile. Au nord les températures sont plus hautes qu'au sud, cela a été donc propice à l'expansion urbaine précoce et plus fortunée.

15 Un exemple de l'actualité, est un mandat de démolition de maisons modestes d'une plage d'Armação, bien qu'étant encore très préservée par le peu d'habitants locaux et considérée comme Aire de préservation culturelle. Ces démolitions vont servir à « nettoyer » l'espace pour la construction d'un complexe hôtelier, un port de plaisance et un téléphérique panoramique. Le projet Matadeiro Resort est destiné au « tourisme écologique », à l'observation de baleines, etc. L'investissement est évalué initialement à 6 millions de dollars américains.

16 Dos Santos Dilma Lucia, Projet Politique Pédagogique (PPP) de l'école, 2010.

17 La notion de quartier à Florianópolis est assez singulière et bien différente de la notion de quartier dans l'espace urbain français. Des caractéristiques relatives à la distance du centre ville, à la spatialité et le nombre d'habitants est assez varié. A Florianópolis le quartier peut être synonyme de plage, pas toujours aménagée pour l'accueil des vacanciers. 
Au sud, le climat plus froid, l'insuffisance de transport, en générant des difficultés de mobilité urbaine ainsi qu'une nature exubérante ont attiré un autre type de composition démographique et économique. Le profil de classe moyenne et inférieure, des gens provenant de presque tout le pays, a joué fortement sur l'expansion tardive de cette communauté. Et un peu près, comme par tous les recoins de l'Ile de Santa Catarina, la formation locale au départ s'est constituée avec les descendants des colonisateurs arrivés vers 1750 et des esclaves, arrivés dans les années suivantes, pour y travailler dans la pêche ou dans l'agriculture. Les descendants des entrepreneurs de l'époque de la chasse à la baleine, d'où vient le nom Armação, ont aussi donné leur contribution à l'histoire de l'occupation du lieu.

Le sud de l'île, dans ces dernières années est la destination d'un grand nombre de militants autour des questions socioculturelles et écologiques. Mais, c'est dans les années 70, 80 et 90, qu'elle a vu arriver les nouveaux arrivants qui iront soit pousser soit freiner le changement local. Ceci a fait naitre des disputes locales entre ceux qui veulent le «développement» local et ceux qui pensent à conserver le District Pântano do Sul comme grand parc municipal, loin de la spéculation immobilière qui submerge l'île. Or, ces gens ont quitté les petites et les grandes villes du sud ou sud-est du Brésil, mais aussi de l'Uruguay et de l'Argentine en quête d'un paradis, d'une vie de simplicité au bord de la mer. Et ils ne veulent pas perdre ce bout de tranquillité conquis.

Le fait est qu'un ensemble d'habitants locaux parmi les nativos, ceux nés dans l'île, et les os de fora, les immigrants, qu'ils soient Brésiliens ou étrangers, de tout niveau de formation et de tout niveau social -des surfeurs, des artistes et artisans, des ouvriers, des fonctionnaires, des professionnels libéraux, des enseignants ou des professeurs universitaires, entre autres-ne veulent pas voir pour le sud de l'île un modèle de développement subi par le nord de l'île, destiné surtout aux classes sociales aisées et au tourisme prédateur.

Ces habitants cherchent à empêcher, par précaution, la transformation de l'île et notamment le sud de celle-ci en une petite Rio de Janeiro au sens de l'accroissement de la population sans l'infrastructure rassurante, en termes d'assainissement basique et de mobilité urbaine; et ceci également, pour épargner la ségrégation de la partie de la population la plus démunie telle que les familles des pêcheurs qui habitent dans des endroits convoités, et encore plus que pour les questions sociales, pour la préservation de l'environnement pour les générations actuelles et futures.

Nous avons une position très claire : freiner l'urbanisation de l'île. Les natifs du Pântano do Sul sont divisés, n'est-ce pas ? Il y en a une partie... qui envisage le progrès et ce progrès c'est la construction, c'est l'asphalte, c'est construire des maisons. Tout cela est progrès. Le progrès classique, ok ? Il y a ceux qui veulent cela, qui soutient cela sous l'allégation que cela produit l'emploi, sous l'allégation que cela va améliorer leurs vies, et des choses 
pareilles. Et, il existe une autre partie de manézinho ${ }^{18}$ qu'on va dire plus respectueux de l'environnement et qui participe à la lutte pour freiner la spéculation, n'est-ce pas ? (Gert Schinke, représentant du Núcleo Distrital do Pântano do Sul, le 21 juillet 2011)

Selon Margaret Pimenta (2005 : 39), le modèle de progrès de développement adopté par les politiques urbaines locales a suivi la logique de rationalisation du propre processus de possession et de valorisation des terres adjacentes aux centres urbains. Cela, en plus d'accentuer les inégalités sociales, contribue à renforcer le sentiment de vulnérabilité des communautés traditionnelles et par conséquent à développer un nouveau profil économique et social de la ville.

$\mathrm{Au}$ centre de toute cette complexité urbaine, persévèrent des projets pour l'espace de l'île conçus dans des réalités distinctes et par des représentations différentes de développement durable. Ceux-ci, engendrent des négociations constantes avec le pouvoir public. D'un côté, on voit l'ambition pour la constante « modernisation » des complexes touristiques avec la construction d'hôtels et d'immeubles avec nombreux étages au bord de la mer, la construction de terrains de golf, des ports de plaisance, de centres commerciaux modernes, par exemple. Et d'un autre côté, on constate la lutte pour la préservation des espaces de transmission culturelle et des espaces de conservation de la nature avec la création de parcs naturels, de pistes cyclables, d'espaces de loisir pour les enfants et jeunes adolescents, etc.

Partagés entre la pression de la modernité et l'effort de " patrimonialisation de l'espace » (Di Méo, 1994 : 17), ceux qui défendent ce dernier entament une lutte pour l'appropriation du territoire et jouent un rôle décisif dans la société locale pour préserver l'identité du lieu et le lien collectif.

\section{Une école engagée avec les questions locales}

L'école Dilma Lucia dos Santos, d'environ 600 élèves, rassemble l'enseignement élémentaire et le collège. ${ }^{19}$ Avec le slogan de l'école « Construire et transformer »

18 Ce mot désigne le surnom des ceux qui sont nés dans l'île de Santa Catarina. Un synonyme de natif. Au Brésil, l'expression manè signifie idiot, lent, stupide, abruti et ainsi de suite. Cependant dans l'île de Santa Catarina le terme, selon le site consulté, vient de l'époque de la colonisation surtout par référence aux Portugais venus des îles de l'archipel des Açores. Al'époque, le prénom Manoel était un des plus utilisés au Portugal. Cela a contribué à désigner les résidents de l'île de « manoelzinhos » (diminutif de Manoel). Cette expression au fil du temps a été transformée en manèzinho puis manè. Ainsi manè est la déformation du nom Manoel. Le même terme alliant une connotation péjorative ailleurs, souvent provoque des quiproquos.

19 Au Brésil, la petite enfance, l'école élémentaire et le premier cycle des études du second degré sont sous la responsabilité des administrations municipales. Ces responsabilités vont de l'entretien du bâtiment, de l'inscription des élèves, de la délibération du programme scolaire au payement des enseignants et des autres fonctionnaires Particulièrement à Florianópolis, jusqu'au début des 
nous pouvons déjà songer à quelques dynamiques de cette école, plus connue dans la ville comme Ecole d'Armação.

Cette école assure un rôle social remarquable dans la communauté avec la promotion d'une diversité d'activités dans et hors ses murs, conçus pour rendre publics les projets culturels et scientifiques des enseignants et les travaux des enfants. Au summum de son intégration avec la communauté, dans les années 1990 et début de 2000 l'école engendrait fortement la participation des familles des élèves dans la préparation des kermesses et dans la décoration des fêtes, organisées aussi pour l'obtention des fonds nécessaires à des petites réparations, des voyages pédagogiques, visites à musées, sorties d'étude et pour l'achat de livres, fournitures, etc.

Parmi les différentes activités pédagogiques ouvertes à la communauté, à caractère culturel et récréatif - ce qui amenait une foule de gens à l'intérieur de l'école, ce dont j'ai témoigné et participé en tant qu'enseignante, il avait la Foire des arts et des sciences, la fête de Saint Jean et le Festival de folklore avec la présentation du Boi-de-mamão, le Bingo de la fête des Mères, etc. D'autres activités étaient réservées aux élèves, mais ne passaient pas inaperçues dans la communauté telles que le championnat de surf et jeux d'été, les cycles des discussions pour les parents, des ateliers de lecture et production de texte, le recyclage, les voyages scolaires dans le département, etc.

Après 2004, l'école, déjà installée dans un nouveau bâtiment, a été bouleversée avec le changement constant de directeurs et d'enseignants. ${ }^{20}$ Ainsi, petit à petit l'école a réduit ses activités " vitrines ». Malgré cela, l'école continue à être un pôle d'attraction et de rencontre de la communauté.

Avec une grande préoccupation tournée vers la culture locale et les questions environnementales, le projet d'école, appelé Projet politique pédagogique (PPP) institue une ligne cohérente de pratiques pédagogiques. Ces pratiques se traduisent dans la formation continue des enseignants, dans des pratiques d'Education environnementale et de sauvegarde de manifestations culturelles comme par exemple les projets d'études sur la mémoire des personnes âgées et des descendants de

années 90, un équipement de petite enfance partageait avec l'école et le collège le même bâtiment ; néanmoins la maternelle avait une direction séparée. Cependant l'école et le collège continuent jusqu'à aujourd'hui à partager non seulement le même bâtiment, mais la même direction et les mêmes horaires, dans une perspective d'intégration et de continuité de l'éducation des enfants et des jeunes adolescents.

20 Actuellement avec les départs en retraite, les arrêts maladies de longue durée, les transferts des enseignants vers d'autres postes à l'administration scolaire, etc., de nombreux enseignants à l'école disposent d'un contrat de travail de type CDD; donc ils ne restent à l'école pas plus d'un ou deux ans. Cela ne permet pas la création d'un lien durable entre l'enseignant, l'école et la communauté, situation néfaste à l'engagement envers les questions locales. 
l'esclavage. L'école accueille les élèves, dans le période opposée aux cours, pour le projet Radio-école Onda jovem. ${ }^{21}$

L'école est ouverte à des activités tournées vers la communauté comme les séances de cinéma, les réunions pour la discussion et construction du Plan directeur $\mathrm{du}$ district, les cours de formation continue, les ateliers d'arts et d'artisanat, entre autres activités culturelle et sportives réalisées pendant le week-end sous le titre d'Ecole Ouverte. ${ }^{22}$ De plus, l'école promeut en juin ou juillet la traditionnelle et réputée Fête de Saint Jean, ${ }^{23}$ avec la participation de l'ensemble de la communauté scolaire et la mise en scène du traditionnel Boi-de-mamão, ${ }^{24}$ très représentatif de la culture de la ville et du littoral de Santa Catarina, est un spectacle assez attendu par tous.

L'école collabore aussi à des projets de recherche institutionnelle, comme celle du Patrimoine historique et artistique national (IPHAN) ainsi qu'à la formation des étudiants universitaires.

Or, tous ces pratiques sociales inscrivent l'école dans une forte tradition dans la localité, dans une relation durable avec la communauté, et définissent son rôle, place et identité malgré sa responsabilité sociale et sa projection dans l'avenir non seulement dans la localité mais davantage dans le District Pântano do Sul. On peut dire que ces pratiques culturelles réalisées à l'intérieur de l'école et ouvertes au public, ${ }^{25}$ ainsi que celles dont l'école est représentée par ses élèves, ${ }^{26}$ cartographient la place de l'école dans le territoire, et de plus lui donnent une importante visibilité dans la région et dans localités voisines, voire l'agglomération toute entière.

21 La Radio Nouvelle Vague voit sa programmation réalisée par des élèves, accompagnés par un enseignant pour les événements qui animent l'école et le quartier, comme des projets de différentes disciplines, des fêtes et même les élections municipales. La radio fonctionne pendant la pose de récréation du matin et de l'après-midi.

22 L'école ouverte est un projet issu du Secrétariat d'éducation de la municipalité.

23 L'objectif de la Fête de Saint Jean, en plus de préserver la culture et la mémoire collective, est de récolter de l'argent pour financier des activités et des projets pédagogiques, étant donné les ressources financières insuffisantes de la municipalité. En même temps, elle est devenue un canal très important de proximité et de dialogue entre l'école et la communauté.

24 Le Boi-de mamão est une manifestation du folklore de l'Etat de Santa Catarina. Composé de plusieurs personnages, hommes et animaux, ceux-ci rentrent en scène dansant. Ils sont accompagnées par de musiciens qui narrent l'histoire de la mort et résurrection d'un bœuf. On trouve cette pratique culturelle dans le nord et Nordeste du Brésil avec différents noms, les plus connus sont Bumba Meu Boi et Boi Bumbá.

25 Pendant les fêtes, l'entrée à l'école n'est pas contrôlée et souvent des personnes d'autres communautés viennent participer de ces occasions.

26 Pendant un de nos séjours en Armação, nous étions présente lors d'une autre grande Fête de Saint Jean (réalisée devant l'église située au cœur de la localité) organisée par le Conseil des habitants et animée par la directrice adjointe de la Fondation Municipale de Culture, ancienne directrice de l'école. En plus des spectateurs (des habitants de la région et alentours et de touristes étrangers), on a observé la présence d'enseignants et d'élèves de l'école qui ont présenté le spectacle Boi-de-mamão. 


\section{Les projets scolaires consacrés à l'environnement}

En ce qui concerne les questions environnementales, bien que tenues au cœur dans le Projet politique pédagogique de l'école, on peut dire que présentement, à partir de nos observations, elles sont plus une directive éducationnelle qu'une « résolution générale » et ceci peut s'expliquer par le changement continu d'enseignants, le manque des temps dédiés à des pratiques d'échange pédagogique ainsi que la spécificité de leurs formations universitaires.

Cependant, même dans ce contexte, l'école possède encore différents projets menés individuellement par les enseignants, ainsi que des projets qui fortifient le collectif de l'école comme celui de la Salle de Recyclage, le Programme de recyclage de papier (Pro-repa) ${ }^{27}$; le Projet «Plantar e Colher: Um jeito de aprender $\aleph^{28} \mathrm{du}$ jardin potager qui est issu d'un projet d'une classe de l'école élémentaire et qui s'est transformée dans un des projets de l'école; les projets écologiques du Laboratoire de sciences et le Projet Entorno Escolar (PEE), que l'on approfondira dans la suite de l'article.

Pendant nos séjours, on a pu observer le travail remarquable d'une enseignante de sciences qui traite les questions environnementales de l'actualité du quartier in situ et développe des activités pratiques sur et dans les différents écosystèmes, dans une approche participative intégrée au territoire.

Une activité annuelle qui engage la totalité de l'école est la Semaine de l'environnement. Pendant ce temps-là, toute l'école se mobilise pour la réalisation d'activités diverses, parmi celles-ci la conscientisation sur des questions relatives à l'environnement comme l'alimentation saine, la production, la réduction et la réutilisation de déchets. Puis, sont réalisées des randonnées et des sorties d'étude de l'environnement dans les parcs écologiques municipaux et dans différents espaces de la région où la nature est préservée.

27 Le Pro-Repa existe à l'école depuis 1993. Les objectifs du programme sont la construction avec la communauté scolaire des habitudes de non gaspillage, du tri de déchets, de réutilisation des objets, de recyclage, de recherche, de communication et de fabrication des différents et variés objets, de manière ludique et créative.

28 Le projet « Planter et récolter: une façon d'apprendre », compte en plus de l'activité avec le potager, la production de plantes en pépinière, le compostage des déchets organiques et le jardinage. Les objectifs du projet sont de permettre aux élèves de comprendre les dynamiques de l'environnement, de promouvoir l'alimentation saine, de participer à la conservation de la nature, et d'élargir les explications sur l'interférence humaine sur celle-ci, et de comprendre le monde et ses transformations. La coordinatrice du projet travaille en partenariat avec les enseignants et avec la cuisine de l'école. 


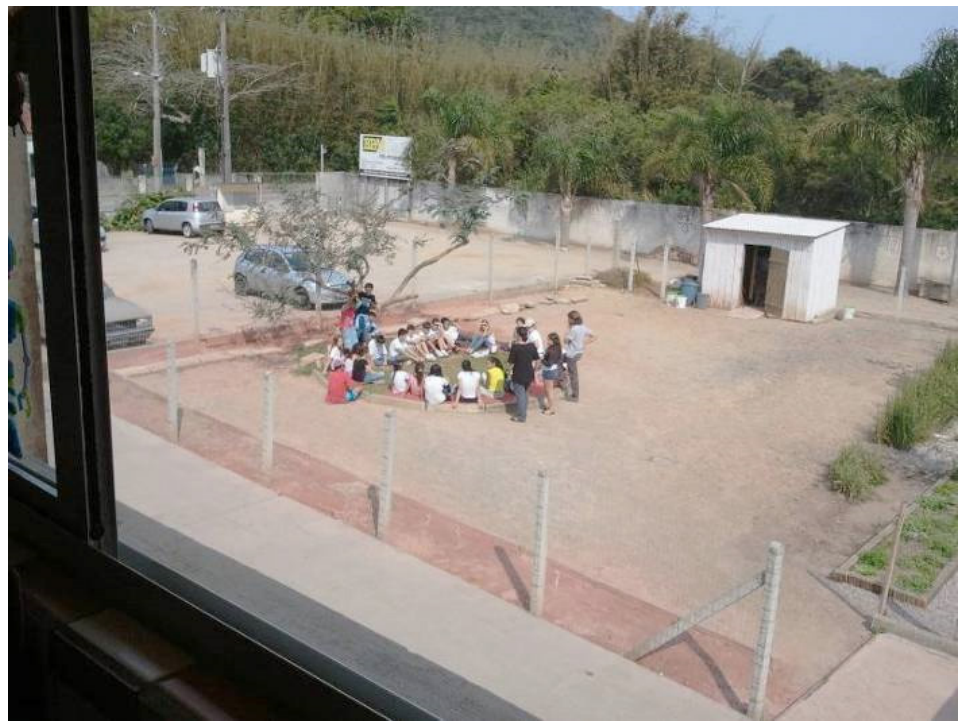

Figure 2. Réalisation du Projet « Plantar e Colher: um jeito de aprende » Photo prise par l'auteur

C'est dans ce contexte que l'école s'inscrit dans les pratiques liées à l'environnement. Par au-delà de ses murs et par le principe de la logique collective, cette école a l'objectif principal de construire une société de plus en plus critique et engagée à l'éthique et aux valeurs de la nature et du groupe social.

\section{Un projet territorial : le projet Entorno Escolar}

Pour comprendre ce territoire, que nous appelons « le territoire de l'école » dont nous parlons ci-dessus, il faut le penser dans l'ensemble de la dynamique espace-temps, indissociés du paysage local qui comprend la plage, l'urbain et le rural. C'est dans ce contexte, dans lequel se déroule le cadre de vie de la communauté d'Armação, un décor d'interactions et d'interconnaissances assez complexes et parfois conflictuelles, qu'un collectif local promeut l'organisation politique et juridique du territoire pour un aménagement soutenable et un développement socio-économique local viable. Dès lors, il s'agit d'une « lutte » pour la justice socio-environnementale (Faburel, 2012).

L'absence de loi qui réglemente les Corridors écologiques au Brésil et particulièrement à Florianópolis, a autorisé la construction en 2004 du bâtiment de l'école Professora Dilma Lúcia dos Santos dans le domaine naturel qu'elle revendique actuellement pour la préservation. En prenant conscience du trésor sous ses pieds, l'école devient l'acteur fondamental vers la patrimonialisation de l'environnement et de la culture locale. 
Ainsi, appuyé sur la législation urbaine et environnementale, le projet Entorno Escolar (expliqué en grandes lignes dans l'introduction), conçu par la communauté scolaire, revendique depuis 2005 auprès de la mairie de Florianópolis 1'expropriation de trois terrains vides situés autour de l'école Dilma Lucia dos Santos, d'à peu près de 100 ha (y compris l'école), qui englobent une diversité d'écosystèmes et de paysages. L'aire configure un Corridor écologique entre deux domaines protégés: le Parc municipal de Lagoa do Péri (2030 ha) et le Parc municipal de Lagoinha do Leste (480,5 ha), qui garantit la liaison entre les écosystèmes marins et côtiers de la Forêt Atlantique.

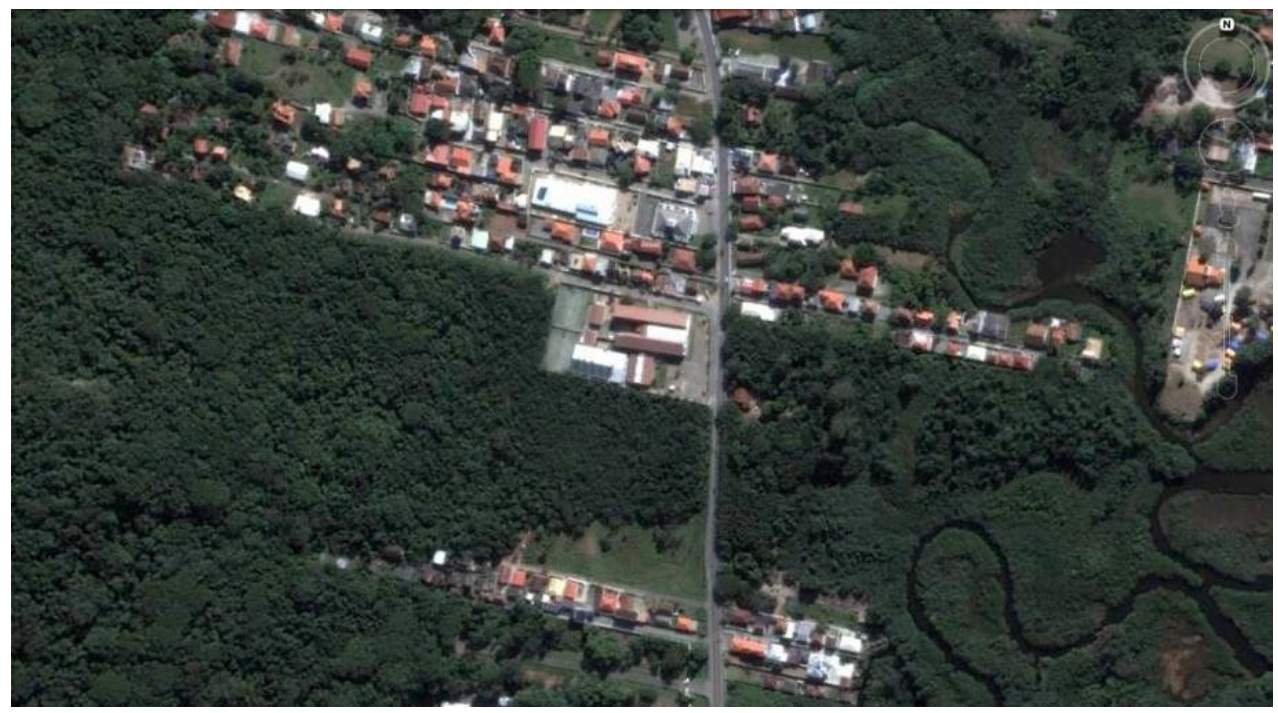

Figure 3. L'école et le corridor écologique vus du haut. Adapté de Google earth de l'auteu

En 2008, après la réalisation d'une pétition et de plusieurs manifestations publiques dans la communauté, le Project Entorno Escolar a été présenté et discuté avec le maire et ses conseillers dans l'amphithéâtre de l'école, ayant la participation de la communauté. Après de nombreuses pressions politiques, la communauté a obtenu, le 6 juillet 2010, la signature du maire dans un décret-loi «provisoire» qui déclare les terrains autour de l'école comme usage public, en conformité avec le décret-loi N. ${ }^{\circ}$ 3365 de 21 de juin $1941,{ }^{29}$ pour satisfaire les aspirations et les besoins collectifs de la population locale, dessinant ainsi le cadre de vie souhaité pour le lieu. Cependant, en 2014, le jeu n'est pas encore gagné car il existe encore de nombreux enjeux pour

29 Ce décret-loi prévoit l'expropriation amicale ou juridique. Source : Diário Oficial do Município de Florianópolis. http://portal.pmf.sc.gov.br/arquivos/diario/pdf/07_07_2010_18.34.31. b055fc807bf4e2736391cd21efbdf8c8.pdf Consulté le 03/11/2015. 
y parvenir, tels que l'achat des terrains, étant donné qu'un projet pour la construction d'un condomínio fechado ${ }^{30}$ a été conçu par leurs propriétaires lorsque qu'ils ont découvert le projet de la communauté, afin d'augmenter la valeur foncière.

Cet espace, vacant de longue date, suscite des questionnements sur son futur incertain et devient le symbole d'un échange de reconnaissance pour une partie de la communauté, pour les propriétaires, qui devront recevoir au moins des compensations pour leurs terrains, ainsi que pour les hommes et des femmes politiques qui bénéficieront en dernier instance des lauriers en cas de concrétisation du projet, représentés souvent au Brésil par une plaque d'inauguration avec le nom du maire de la ville. Cela compose un système que Mauss (1985: 171), appelle l'échangedon en constituant un lien entre l'individuel et le collectif.

Discuter les problématiques du lieu de vie, participer et délibérer au-delà de la structure et de l'organisation de l'école, impliquent l' « intérêt » aux dynamiques de long terme et l'« engagement » (Becker, 2006; Zask, 2011) collectif territorial. Mais aussi la reconnaissance (Fraser, 2004) socio-environnementale et politique d'un collectif par les hommes politiques, ainsi que le respect envers les textes constitutionnels dans les politiques durables d'aménagement.

Le récit suivant nous montre une lecture de la réalité locale; la liaison entre quotidien et lieu de vie s'unit pour construire un projet social dans le cadre de l'école.

La communauté veut du gouvernement le don d'un terrain pour l'amélioration de la communauté et non pour le commerce. Il existe un espoir que la mairie achète les terrains et qu'elle fasse un don à la communauté pour le bénéfice des jeunes. [...] Il n'a plus beaucoup de poissons dans la mer, donc la pêche n'est plus rentable. [...] Deux années en arrière, le 7 Septembre (Fête de l'Indépendance du Brésil), on a fait dans les rues du quartier un défilé, une manifestation sur la question de l'environnement et on a fini avec « abraço na escola $\|{ }^{31}$ et de ses environs. La communauté a participé très activement, comme toujours. Mais maintenant tout est en standby à cause de questions politiques. Ces questions interviennent négativement dans nos projets, ils empêchent l'avancement et l'obtention de bons résultats. La mobilité des enseignants rend aussi difficile leur engagement dans le projet. On veut faire un musée et une Ecole d'éducation environnementale qui soit d'avantage destinées aux enfants, où l'on puisse développer des projets qui répondent aux besoins des jeunes. [...] Je voudrais que le gouvernement ait un regard dirigé vers la communauté, qu'il construise un stade couvert à côté de l'école, un gymnase dans le quartier, un parc avec beaucoup d'arbres et des bancs, etc. [...] Le programme d'éducation de la mairie prévoit la connaissance du quartier et j'estime beaucoup cette directive, car cela nous donne de l'initiative et nous motive. L'axe du notre programme scolaire, c'est l'Etude du Milieu. L'objectif est de faire que les élèves s'approprient le contexte où ils vivent de façon critique. Tout cela est très lent, mais on a des réponses : un de ces jours précédents, une élève de l'« Escola Técnica », notre ancienne élève, est venue à l'école pour nous demander une vidéo en VHS

30 Désigne une sorte de « quartier chic » privatisé, tel qu'un club privé, dont l'accès et l'utilisation des places, des parcs et des voies, sont contrôlés.

31 Une ronde humaine faite autour de l'école et d'un des terrains. 
du Rio Sangradouro. L'espoir est que les uns et les autres s'intéressent aux questions de l'environnement du quartier. Ici c'est la « Serra do Mar »; on veut préserver ce qu'on a. Tu sais, la construction des lotissements en direction au Pântano do Sul ont été arrêtés. Dans le Plan directeur d'Armação, existe la participation de la communauté et les habitants utilisent celui-ci pour éviter la déprédation de l'environnement local. Tu sais qu'il en a des toucans qui survolent l'école ? Le Plan directeur est formé pour les leaders de la communauté. Le gouvernement a créé un problème car ces personnes luttent pour les questions de la communauté. La communauté est puissante, ici tout est discuté car les personnes veulent tout discuter, mas rien n'est fait sans le Plan Directeur, ça c'est un souci ! (Extrait du récit d' ltair lves Lúcio Felipe spécialiste d'orientation scolaire, le 8 mars 2010)

Dans ce récit, la reconnaissance apparait en tant que demande de type territoriale et environnementale sur laquelle le développement continu rend possible l'existence des habitants et des jeunes en dehors des drogues et de la marginalisation. La reconnaissance constitue un complément de théories de la démocratie (Zask, 2011 : 291). Le souhait d'un don notamment du pouvoir public est une étape indispensable dans la négociation de l'école au nom de la communauté, et donc de reconnaissance. Dans le raisonnement de notre interlocutrice, en connaissance de cause, ces terrains seront « garantis » pour le projet Entorno Escolar seulement avec leur insertion dans le dispositif municipal d'occupation du sol, le Plan directeur d'urbanisation de la ville, en liaison avec la participation et la lutte communautaire. Cependant on voit que la municipalité n'est pas intéressée par ce projet car elle l'a écarté du Plan directeur de la ville approuvé début 2014.

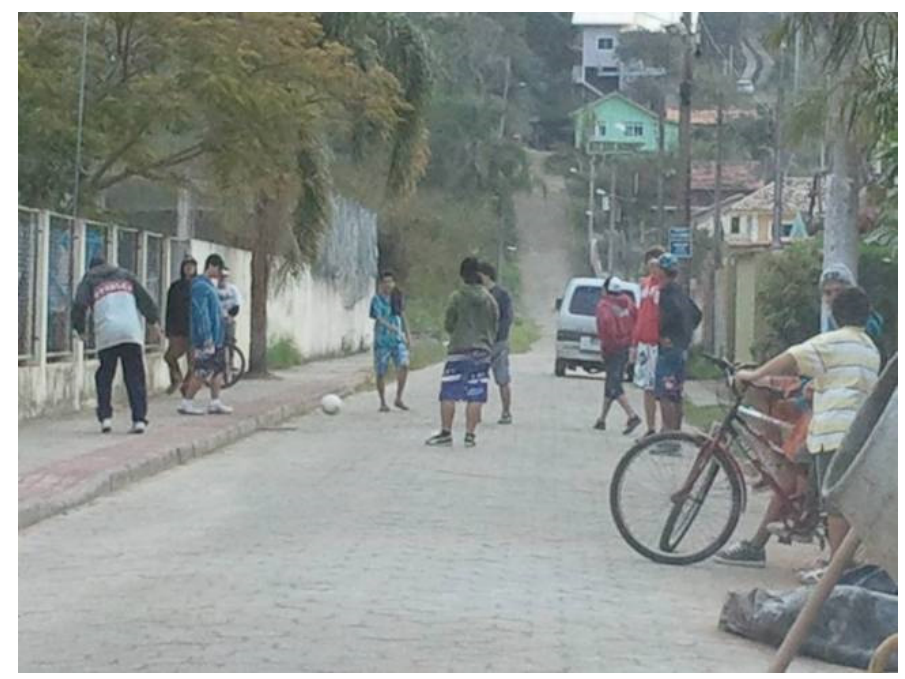

Figure 4. Des adolescents en train de s'amuser devant le portail d'entrée de l'école. Photo prise par l'auteur 
La principale évolution de ces échanges est de donner un nouveau sens à un territoire qui reste attaché à une identité culturelle pour qu'il puisse atteindre un niveau supérieur de développement socio-culturel. Cet aspect combine à la fois intégration sociale et créativité individuelle. De plus, il accroît les attributs moraux de la communauté puis rend possible l'acquisition d'une visibilité sociale particulière, tournée vers la patrimonialisation de la richesse environnementale et paysagère tout en se préservant des modèles modernes d'urbanisation et occupation durables des côtes en voie d'internationalisation. Cela est un véritable enjeu pour les propriétaires et les hommes politiques car il englobe un éventail d'aspects tels que pouvoir, argent, valeurs morales et éthiques.

\section{Conclusion : les expériences participatives comme levier pour la préservation de la nature}

Le développement et la réorganisation des territoires sont des indicateurs du processus de modernisation des villes. Horizon de projections politiques et économiques, de nouveaux projets forgent l'utopie urbaine notamment à Florianópolis à travers de la construction de nouvelles routes, de l'occupation des espaces dans la ville -d'où l'on peut plus contempler la nature-, par de centres commerciaux, Resorts de luxe, terrains de golf, etc.

Si les normes et les politiques de développement et d'aménagement urbain actuelles reflètent les modèles d'organisation d'une époque, elles simplifient souvent la complexité de la réalité. Celles-là mettent en danger le cadre de l'environnement en faveur la mise en avance la modernité, conçue en tant que «synonyme» de qualité de vie et liée directement à l'appropriation du concept de développement durable basé sur la construction de bâtiments énergétiquement propres et sur la réalisation d'activités écologiques et anti-stress pour les entreprises du bâtiment et du tourisme de haut niveau, résultant courant dans la ségrégation socio-environnementale.

$\mathrm{Au}$ Brésil, dans le contexte historique de ces dernières années « post-constitution », on voit d'un côté l'avancement de la participation démocratique et de l'autre les difficultés que revêt l'expérience de la participation populaire, notamment à Florianópolis.

Les enjeux qui entourent la question foncière et l'environnement créent des énormes défis dans le cadre de la réalisation de projets collectifs d'intérêt public et provoquent souvent un sentiment de manque d'autonomie, d'impuissance et de non-reconnaissance publique dans le cadre des revendications ainsi que d'injustice sociale, culturelle et environnementale. De plus, les luttes entamées dans un cadre de démocratie participative sont ordinairement attachées aux stratégies politiques de délégation de pouvoir, inhérentes à la démocratie représentative, ce qui retarde le processus. 
Dans ce contexte, l'école et le territoire -connectés par des expériences sociales et affectives, individuelles et collectives et inextricablement liées dans le processus de croissance et d'aménagement de la vill-s'influencent mutuellement dans le cadre de la réorganisation des espaces et dans la génération de nouvelles situations et actions. Et l'école a un rôle non négligeable dans l'émancipation sociale, dans la participation démocratique et dans l'établissement d'un réseau social d'influence territoriale pour la valorisation et la préservation du patrimoine culturel et naturel ainsi que pour l'agencement urbain local.

De toute évidence, l'anthropologie de l'environnement doit aller au-delà des questions liées à la culture et à la nature. Les acteurs et groupes sociaux interrogés à partir d'une localisation géographique précise et de rapports qui entretiennent avec leur espace vécu et le pouvoir, témoignent une diversité d'interprétations, d'usages et de pratiques envers l'environnement. Et dès lors révèlent leurs façons de faire société. A cet égard, l'anthropologue engagé dans la lutte pour la justice socio-environnementale devient incontournable dans les pratiques démocratiques et conflits fonciers, ainsi que dans l'amélioration du développement et de la gestion urbaine durable.

Toutes ces questions expriment, d'un côté, un défi pour les acteurs sociaux en termes de responsabilité et d'implication individuelle et collective, qui est au cœur de la démocratie participative et du combat envers les injustices socio-environnementales. Et de l'autre côté, existe le devoir des hommes et femmes politiques de prendre en compte dans les politiques urbaines la participation démocratique en tant que forme de reconnaissance de la diversité des modes de vie, ainsi qu'au droit des populations à des villes de qualité environnementale et paysagère.

Pour faire avancer le débat, signalons que la Fondation municipale de l'environnement de Florianópolis (FLORAM) ${ }^{32}$ a récemment réalisé une consultation publique ${ }^{33}$ dans l'amphithéâtre de l'école pour la réévaluation des limites physiques des aires de préservation du Parc municipal de Lagoinha do Leste. L'un des objectifs de la Fondation est d'obtenir des ressources financières auprès du Ministère de l'Environnement ainsi que de viabiliser le corridor écologique qui intègre l'écosystème du parc voisin de Lagoa du Péri, ce qui pourrait contribuer à avancer le projet de l'école.

En tout cas, ces éléments constituent un pas fondamental dans la justice socio-environnementale, aussitôt pour la transformation historique de l'occupation du sol, des conditions de vie et d'habitat, ainsi que pour l'évolution de la pensée, des règlements juridiques et des systèmes politiques. Ainsi devient-il urgent de fai-

32 Cette fondation est responsable de la politique environnementale de la municipalité, donc responsable entre autres de l'implémentation, l'inspection et l'administration des aires municipales protégées.

33 http://www.pmf.sc.gov.br/entidades/floram/index.php?pagina=notpagina\&noti=14499 Consulté le $03 / 11 / 2015$. 
re évoluer les pratiques d'émancipation des sociétés contemporaines à des niveaux local et mondial et dès lors l'évolution des modèles urbains actuels.

\section{Bibliographie}

Alexandre, Agripa (2003). Políticas de resolução de conflitos socioambientais no Brasil: o papel do Ministério Público e dos movimentos ambientalistas na Ilha de Santa Catarina. Editora da UFSC e EdiFurb, Florianópolis/Blumenau.

Bacqué, Marie-Hélène (2005). "Action collective, institutionnalisation et contre-pouvoir: action associative et communautaire à Paris et à Montréal”. En: Espaces et sociétés, N. ${ }^{\circ} 123$, pp. 69-84.

Becker, Howard (2006). "Notes sur le concept d'engagement”. En: Revue de Sciences humaines, vol. 11, pp. 177-192.

Cefaï, Daniel (2010). “L'engagement ethnographique”. En: L'Engagement ethnographique, Éditions de l'École des hautes études en sciences sociales, Paris, pp. 7-21.

Chaui, Marilena (2006). Brasil: mito fundador e sociedade autoritária. Editora Fundação Perseu Abramo, São Paulo.

Dewey, John (2010). Le Public et ses problèmes, Gallimard, coll. Folio Essais, 2010. Traduit et présenté par Joëlle Zask [The Public and its Problems, 1927]

Di Méo, Guy (1994). "Patrimoine et territoire, une parenté conceptuelle". En: Espaces et sociétés, N. 78, Éd. Érès, pp.15-34.

Dos Santos, Dilma Lucia (2010). Projet Politique Pédagogique (PPP) de l'école.

Faburel, Guillaume (2012). "La ville durable aux défis des injustices environnementales, Constats empiriques et enjeux sociopolitiques". En: Flux, N. ${ }^{\circ}$ 89-90, pp. 15-29.

Fantin, Márcia (2000). Cidade dividida: Dilemas e Disputas Simbólicas em Florianópolis, Cidade Futura, Florianópolis.

Fraser, Nancy (2004). "Justice sociale, redistribution et reconnaissance". En: Revue du MAUSS, N. ${ }^{\circ}$ 23, pp. 152-164.

Gausset, Quentin (2003). “Séminaire de l'Association française des anthropologues. Actualités de l'Anthropologie". En: Journal des anthropologues, pp.94-95.

Lago, Paulo Fernando (1996). Florianópolis: A Polêmica Urbana, Fundação Franklin Cascaes/ Palavra Comunicação, Florianópolis.

Machado, Ewerton (2000). Florianópolis. Um lugar em tempo de globalização, FFLCH/USP, Tese de Doutorado, São Paulo.

Maricato, Ermínia (2013). "É a questão urbana, estúpido!" En: Cidades Rebeldes. Passe livre e as manifestações que tomaram as ruas do Brasil. Ed. Boitempo, São Paulo, pp. 32-45

Mauss, Marcel (1985). "Essai sur le don. Forme et raison de l'échange dans les sociétés archaïques". En: Sociologie et anthropologie, Les Presses universitaires de France, Paris, pp. 154-171.

Milton, Kay (1997). "Ecologies: anthropologie, culture et environnement". En: Revue Internationale des Sciences Sociales, 154, pp. 519-538.

Pimenta, Margareth (org.) (2005). Florianópolis do outro lado do espelho. Editora da UFSC, Florianópolis.

Pozzobom, Vicente y Rocha, Chico (2013). "Vidéo do Núcleo Distrital do Pântano do Sul".

Zask, Joëlle (2011). Participer. Essai sur les formes démocratiques de la participation. Le Bord de l'eau, Paris. 